\title{
AVALIAÇÃO DO CONSUMO DE SÓDIO EM DUAS UNIDADES DE ALIMENTAÇÃO E NUTRIÇÃO DO RIO GRANDE DO SUL
}

\author{
EVALUATION OF SODIUM CONSUMPTION IN TWO UNITS OF FEEDING \\ AND NUTRITION OF RIO GRANDE DO SUL
}

\begin{abstract}
Alicia Macagnan Alessio, Stéfanie Simonis Lucca, Juliana Paula BruchBertani*.
\end{abstract}

Universidade do Vale do Taquari, Lajeado, RS, Brasil.

*julianapb@univates.br

Recebido em: 09/04/2019; Aceito em: 04/12/2020.

\section{RESUMO}

A pesquisa, realizada em duas Unidades de Alimentação e Nutrição (UAN), pretendeu avaliar o consumo de sódio extrínseco dos comensais preconizado pelo Programa de Alimentação do Trabalhador (PAT). Analisou-se a quantidade de sal utilizada na ficha técnica, nas preparações das refeições e a quantidade de sal consumida nos saleiros. Comparou-se os valores de sódio entre as duas UANs, analisando a diferença entre o sódio estimado na ficha técnica em relação às preparações e ao sódio utilizado nos saleiros e comparados com valores de recomendações estabelecidos pelo PAT. Verificou-se diferença entre as duas UANs: sódio consumido no saleiro, sódio utilizado nas preparações, sódio estimado na ficha técnica e sódio das preparações somados com o sódio do saleiro. Na UAN 1, observou-se valores de sódio superiores nas preparações quando comparadas à ficha técnica, 1928,4 mg vs 1217,6 mg, respectivamente. Em ambas unidades, os valores da ficha técnica foram superiores à referência, $1217,6 \mathrm{mg}$ para UAN 1 e 3112,4 mg para UAN 2, enquanto que os valores do saleiro foram inferiores à referência, $217,6 \mathrm{mg}$ para UAN 1 e 283,2 mg para UAN 2. As unidades apresentaram consumo de sódio elevado, o que pode promover o desenvolvimento de doenças cardiovasculares. Visto que as duas unidades apresentaram resultados significativamente altos em relação à quantidade de sódio oferecida nas refeições, faz-se necessário reduzir o sal das preparações a fim de ofertar uma alimentação mais saudável.

Palavras-chave: Alimentação Coletiva. Nutrição. Sal. Sódio.

\section{ABSTRACT}

The research, carried out in two Food and Nutrition Units (FNU), aimed at evaluating the extrinsic sodium consumption of the diners in the lunch meals, comparing the results with each other and with the one recommended by the Worker Feeding Program (WFP). The amount of salt used in the datasheet, the preparation of meals and the amount of salt consumed in the salt shakers was analyzed. The sodium values between the two FNUs were compared by analyzing the difference between the estimated sodium in the datasheet in relation to the preparations and the sodium used in the salt shakers and 
compared with the values of recommendations established by the WFP. There was a difference between the two FNUs: sodium consumed in the salt shaker, sodium used in the preparations, estimated sodium in the datasheet and sodium of the preparations added with the sodium of the salt shaker. In FNU1, higher sodium values were observed in the preparations when compared to the menu, $1928.4 \mathrm{mg}$ vs $1217.6 \mathrm{mg}$, respectively. In both units, the values of the datasheet were higher than the reference, $1217.6 \mathrm{mg}$ for FNU1 and $3112.4 \mathrm{mg}$ for FNU2, while the values of the salt shaker were lower than the reference, $217.6 \mathrm{mg}$ for FNU1 and 283, $2 \mathrm{mg}$ for FNU2. Units had elevated sodium intake, which may promote the development of cardiovascular disease. Since the two units presented significantly higher results in relation to the amount of sodium offered in meals, it is necessary to reduce the salt of the preparations in order to offer a healthier diet.

Keywords: Collective Feeding. Nutrition. Salt. Sodium.

\section{INTRODUÇÃO}

Segundo a Organização Mundial da Saúde (OMS) (WHO, 2016), o aumento da produção de alimentos processados, a rápida urbanização e a mudança de estilos de vida estão modificando os padrões alimentares da população. As escolhas por alimentos mais densos em energia, ricos em gorduras saturadas, gordura trans, açúcar e sal aumentam visivelmente. O sal é a principal fonte de sódio, onde seu elevado consumo está diretamente associado ao desenvolvimento de doenças, principalmente de origem cardiovasculares (WHO, 2016; MARTINS et al., 2020). Apesar das melhorias no diagnóstico e tratamento, as doenças cardiovasculares ainda são responsáveis pela redução da qualidade de vida e por contribuir para o agravamento de doenças, sendo uma das principais causas de morbidade e mortalidade, representando cerca de $30 \%$ dos óbitos mundialmente em países em desenvolvimento, como o Brasil (TURNER et al., 2017; BRASIL, 2018; MELLO et al., 2020).

De acordo com a Pesquisa de Orçamentos Familiares 2017-2018, sobre a análise do consumo alimentar pessoal no Brasil em relação à ingestão de sódio mostrou valores acima do limite aceitável, sendo referida por $53,5 \%$ da população. $O$ consumo de pizza, carnes processadas, salgadinhos industrializados, biscoitos recheados e refrigerante está diretamente relacionado ao consumo elevado de sódio, e de acordo com o Instituto Brasileiro de Geografia e Estatística (IBGE), foi verificado o consumo superior em adolescentes do que em adultos e idosos nos anos de 2017 e 2018 (IBGE, 2018). Nas Unidades de Alimentação e Nutrição (UAN) o consumo de sódio pelos comensais também é alarmante. No estudo de Borges (2015) verificou-se uma elevada ingestão de sódio consumido apenas na refeição do almoço, chegando a uma média de 2927,12 mg (BORGES, 2015).

O sódio pode ser encontrado nos alimentos in natura, no sal adicionado ao preparo dos alimentos, no uso do saleiro à mesa e principalmente nos alimentos industrializados, sendo ampla sua oferta. O cloreto de sódio tem várias funções em alimentos processados: adiciona sabor e estrutura, além de 
preservar e aumentar a ligação da água às proteínas da carne e peixe (STEFFENSEN et al., 2018). No entanto, evidências mostram que há associação do consumo elevado de sódio com o desenvolvimento de diversas doenças crônicas não transmissíveis (DCNT), como hipertensão arterial, doenças cardiovasculares, acidente vascular cerebral, hipertrofia ventricular esquerda, doenças renais e câncer de estômago. A redução no consumo de sal tem sido identificada como uma das intervenções mais custo-efetivas para reduzir a carga de DCNTs (OLIVEIRA et al., 2015).

Devido à crescente preocupação da população em relação à praticidade no preparo dos alimentos, a demanda por refeições prontas para o consumo favoreceu o aumento do número de estabelecimentos do setor de alimentação fora do lar e diversificou seus serviços, com destaque para o crescimento no número de UANs (BEZERRA et al., 2017). Estas unidades são responsáveis pela preparação e fornecimento de refeições equilibradas em nutrientes e com qualidade higiênica-sanitária. Desta forma, torna-se relevante pensar em ações dirigidas para esses estabelecimentos em relação à redução do consumo de sódio minimizando o desenvolvimento de agravos à saúde do trabalhador (BRITO; SPINELLI 2016; OLIVEIRA; CARVALHO, 2016).

Tendo em vista a importância do acesso ao consumo de uma refeição saudável e equilibrada, a elaboração de cardápios nas UANs deve ser de responsabilidade do profissional nutricionista, que trabalha com a adequação nutricional das refeições oferecidas por estes estabelecimentos, proporcionando maior segurança alimentar e oferta adequada de nutrientes (LOPES et al., 2016).

Com o objetivo de melhorar as condições nutricionais dos trabalhadores, em 1976 criou-se o Programa de Alimentação dos Trabalhadores (PAT), responsável por estabelecer valores de referência para ingestão de nutrientes (BRASIL, 2020). O PAT tem como principal objetivo fornecer uma melhora na condição nutricional do trabalhador e promover saúde por meio de uma alimentação saudável. Visa ainda uma melhora na qualidade de vida e aumento da produtividade, além de prevenir doenças relacionadas ao trabalho. Este programa, estruturado na parceria entre Governo, empresa e trabalhador, tem como unidade gestora o Departamento de Segurança e Saúde no Trabalho da Secretaria de Inspeção do Trabalho (PEREIRA et al., 2014; MAZZON et al., 2016). Determina que as refeições principais (almoço/jantar/ceia) devem assegurar a quantidade de 720 a 960 mg de sódio (BRASIL, 2006).

Tendo em vista o elevado consumo de sódio e sua relação com as doenças cardiovasculares, o presente trabalho teve como objetivo avaliar 0 consumo de sódio extrínseco em duas UANs do Rio Grande do Sul.

\section{MATERIAIS E MÉTODOS}

Trata-se de um estudo transversal realizado em duas UANs do Rio Grande do Sul, no período de 31 de agosto a 14 de setembro de 2018 a fim de avaliar a quantidade de sal extrínseco consumida pelos comensais. Durante 10 dias úteis, considerando somente o turno do almoço, analisou-se a quantidade de sal calculada nas fichas técnicas das preparações, a quantidade de sal consumida pelos comensais a partir da quantidade inserida nas preparações das refeições e no saleiro exposto no buffet. Ainda, avaliou-se o consumo de sódio 
a partir da soma do sódio consumido nos saleiros com o sódio proveniente das preparações das refeições. Posteriormente, comparou-se o sódio estimado nas fichas técnicas com valores de recomendações estabelecidos pelo PAT, correspondente a 720-960mg per capita nas refeições principais (almoço/jantar/ceia).

O sal nos recipientes (saleiros) do buffet e da produção das refeições foi pesado antes e ao final do expediente, subtraindo a quantidade inicial de sal pela quantidade final. Avaliou-se o sal adicionado nas seguintes preparações: arroz, feijão, guarnições e carnes. As saladas foram excluídas, uma vez que são preparadas e servidas sem temperos. Não foi considerado o sódio intrínseco dos alimentos.

O cálculo per capita foi realizado através da quantidade de sal utilizado dividido pelo total de refeições servidas em cada dia. Considerando que cada 1 grama de sal corresponde a $400 \mathrm{mg}$ de sódio, fez-se um cálculo para obter a quantidade de sódio consumido em miligramas.

Os dados foram analisados através de tabelas, gráficos, estatísticas descritivas e pelos testes estatísticos, teste $t$-student para comparação entre as amostras. Os resultados foram considerados significativos a um nível de significância máximo de $5 \%(p<0,05)$ e o software utilizado para esta análise foi o SPSS versão 22.0.

\section{RESULTADOS E DISCUSSÃO}

A partir das análises de dados, verificou-se diferença significativa entre as UANs em relação à média do consumo de sódio provindo do uso do saleiro, tendo um consumo maior pelos comensais da UAN1 $(p<0,05)$. Também verificou-se diferença na média do sódio utilizado nas refeições, onde a UAN2 utiliza maior quantidade do mineral para a preparação das mesmas, e ainda, na UAN2 o consumo de sódio pelos comensais a partir da soma dos valores encontrados na preparação com o sódio adicional do saleiro foi maior em relação à UAN1 $(p<0,05)$. Verificou-se significância também, nos valores de sódio descritos nas fichas técnicas das preparações, onde a UAN2 apresenta valor superior em relação à UNA1 $(p<0,05)$. Os valores de média de sódio $(\mathrm{mg})$ estão descritos e representados na Tabela 1.

Verificou-se que apenas a UAN 1 apresentou diferença significativa para seus valores $(p=0,037)$. Na UAN 1 observou-se valores de sódio significativamente superiores à UAN 2 nas preparações quando comparadas às fichas técnicas, respectivamente. Demais dados estão descritos na Tabela 2.

A tabela 3 descreve a comparação entre o sódio consumido nas preparações e o sódio estimado na ficha técnica com os valores de referência estabelecidos pelo PAT. Verificou-se que todas as variáveis apresentam diferença significativa para seus valores de sódio quando comparados aos valores de referência. Observa-se que para a quantidade de sódio consumida nas preparações, tanto a UAN 1 quanto a UAN2, apresentaram valores significativamente inferiores à referência. Já para os valores do sódio estimados na ficha técnica, estes foram significativamente superiores à referência em ambas as UANs, principalmente na UAN $2(p=0,005)$. 
Tabela 1 - Valores de sódio (mg) encontrados nas preparações das refeições, nas fichas técnicas e saleiro nas duas Unidades de Alimentação e Nutrição

\begin{tabular}{lcccc}
\hline Variável & UAN & Média & DP & p \\
\hline Sódio saleiro (per capita) & UAN 1 & 217,6 & 63,5 & $0,003^{* *}$ \\
& UAN 2 & 283,2 & 87,4 & \\
\hline Sódio preparações (per capita) & UAN 1 & 1928,4 & 1263,1 & $0,002^{* *}$ \\
& UAN 2 & 2910,8 & 504,8 & \\
\hline Sódio ficha técnica (per capita) & UAN 1 & 1217,6 & 75,5 & $0,000^{* *}$ \\
& UAN 2 & 3112,4 & 839,3 & \\
\hline Sódio preparação + buffet (per capita) & UAN 1 & 2146,0 & 1275,1 & $0,002^{* *}$ \\
& UAN 2 & 3196,8 & 496,5 & \\
\hline
\end{tabular}

Notas: DP - Desvio Padrão; **significativo p $\leq 0,05$; Teste t-student.

Fonte: os autores.

Tabela 2 - Comparação entre os valores de sódio $(\mathrm{mg})$ encontrados na ficha técnica do cardápio e quantidade de sódio encontrada nas preparações das refeições.

\begin{tabular}{llllcc}
\hline Local & Variável & N & Média & DP & P \\
\hline UAN 1 & Sódio ficha técnica (per capita) & 10 & 1217,6 & 75,5 & $0,037^{*}$ \\
& Sódio preparações (per capita) & 10 & 1928,4 & 1263,1 & \\
\hline \multirow{2}{*}{ UAN 2 } & Sódio ficha técnica (per capita) & 10 & 3112,4 & 839,3 & $0,285^{\mathrm{NS}}$ \\
& Sódio preparações (per capita) & 10 & 2910,8 & 504,8 & \\
\hline \multirow{2}{*}{ Geral } & Sódio ficha técnica (per capita) & 20 & 2165,0 & 1131,9 & $0,433^{\mathrm{NS}}$ \\
& Sódio preparações (per capita) & 20 & 2419,6 & 1063,2 & \\
\hline
\end{tabular}

Notas: DP - Desvio Padrão; NS - não significativo; *significativo $p \leq 0,05$, Teste tstudent.

Fonte: os autores.

Segundo a Portaria Interministerial № 66, de 25 de agosto de $2006^{11}$, entende-se por alimentação saudável, o direito humano a um padrão alimentar adequado às necessidades biológicas e sociais dos indivíduos, respeitando os princípios da variedade, da moderação e do equilíbrio, dando-se ênfase aos alimentos regionais e respeito ao seu significado socioeconômico e cultural, no contexto da Segurança Alimentar e Nutricional. Nesse sentido, as UANs desempenham papel importante no que diz respeito ao fornecimento de refeições, pois têm como principal objetivo ofertar uma alimentação nutricionalmente adequada, podendo contar com uma política pública de alimentação direcionada aos trabalhadores, o PAT (BRASIL, 2006). 
Tabela 3 - Comparação entre os valores de Sódio (mg) encontrados nas preparações e quantidade de sódio encontrado na ficha técnica com os valores de referência do Programa de Alimentação do Trabalhador

\begin{tabular}{lcccc}
\hline & Média & DP & Referência & p \\
\hline UAN 1 & & & & \\
Sódio preparações (per capita) & 217,6 & 63,5 & $720-960 \mathrm{mg}$ & $0,005^{\star *}$ \\
Sódio ficha técnica (per capita) & 1217,6 & 75,5 & $720-960 \mathrm{mg}$ & $0,005^{\star \star}$ \\
\hline UAN 2 & & & & \\
Sódio preparações (per capita) & 283,2 & 87,4 & $720-960 \mathrm{mg}$ & $0,005^{\star *}$ \\
Sódio ficha técnica (per capita) & 3112,4 & 839,3 & $720-960 \mathrm{mg}$ & $0,005^{\star *}$ \\
\hline
\end{tabular}

Notas: DP - desvio padrão; **significativo $p \leq 0,01$, Teste t-student.

Fonte: os autores.

De acordo com as exigências nutricionais estabelecidas pelo PAT, as principais refeições devem conter de 600 a 800 calorias, admitindo-se um acréscimo de $20 \%$ (quatrocentas calorias) em relação ao Valor Energético Total -VET de 2000 calorias por dia. O cardápio deverá conter $60 \%$ da composição de carboidratos, $15 \%$ de proteínas, $25 \%$ de lipídios e $<10$ gramas de gorduras saturadas. Ainda deverá conter de 7 a 10 gramas de fibras e de 720 a 960 miligramas de sódio, sendo que o valor diário de referência para o consumo de sódio é de 2400 miligramas (BRASIL, 2006).

Segundo a OMS (WHO, 2016), o sódio é um nutriente essencial necessário para a manutenção do volume plasmático, equilíbrio ácido-base, transmissão de impulsos nervosos e função celular normal. No entanto, o excesso desse mineral está ligado a resultados adversos à saúde, incluindo aumento da pressão arterial, sendo que os principais contribuintes para o consumo de sódio na dieta dependem do contexto cultural e dos hábitos alimentares de uma população.

O presente estudo encontrou um consumo adicional de sódio, tanto pelas preparações oferecidas aos comensais, quanto ao uso do saleiro de mesa, superior na UAN 2, entretanto, verifica-se um consumo expressivo em ambas unidades. Estudo realizado por Conceição et al. (2016) avaliou o consumo de sódio nas preparações de comensais e verificou que os valores excederam as recomendações diárias, e ainda, verificou-se a presença do saleiro nas mesas sendo, na maioria das vezes, utilizados na salada e na própria refeição consumida, desta forma elevando os valores encontrados no estudo. Assim, é aconselhável que o sal seja retirado da mesa para reduzir o consumo de cloreto de sódio na alimentação a fim de evitar seu consumo adicional (CONCEIÇÃO et al., 2016).

Em relação à avaliação do sódio nas fichas técnicas em comparação ao PAT, verificou-se que em ambas as UANs os valores foram superiores às recomendações do Programa, corroborando com os achados de Baltazar et al. (2019), que avaliou o valor médio de sódio dos cardápios e também encontrou 
valores superiores à recomendação do PAT em mais de 90\% (BALTAZAR et al., 2019). Avaliando preparações de 15 restaurantes de São Paulo, Spinelli, Kawashima e Egashira (2011) estudaram valores de sódio em preparações de arroz branco, feijão e carne ofertados aos comensais e verificou-se que o teor de sódio excedeu em $25 \%$ a quantidade máxima recomendada para uma única refeição (SPINELLI; KAWASHIMA; EGASHIRA 2011).

O sódio inserido nas preparações das UANs é um fator preocupante também verificado nos resultados do estudo de Lagemann e Fassina (2015), em que se encontrou uma média de consumo de 2886,49 mg $\pm 830,89$, excedendo, significativamente a determinação do PAT (LAGEMANN; FASSINA, 2015). Demais estudos também encontraram valores superiores avaliando fichas técnicas (CARNEIRO; MOURA; SOUZA, 2013), cardápios de dois restaurantes e de UANs (SALAS et al., 2009; PEREIRA; SOUZA; RIBEIRO 2011), sendo todas cadastradas no PAT. Desta forma, o consumo excessivo de sódio na dieta pode levar ao desenvolvimento de doenças cardiovasculares, além de estar intimamente relacionado à hipertensão arterial (LAGEMANN; FASSINA, 2015).

A incidência de hipertensão no Brasil chega a 20\%, e estima-se que o consumo médio de sal, por dia, seja de 9,6 gramas por pessoa. Estudos indicam que mais de $70 \%$ da população ingere valores superiores ao valor máximo de consumo tolerável para o sódio, sendo o consumo de $89 \%$ homens e $70 \%$ de mulheres. As idades entre 19 a 59 anos consomem mais do que as acima dos 60 anos, ficando em excesso de consumo $80 \%$ e $62 \%$ destas populações, respectivamente (OTTONI; SPINELLI, 2014).

Em estudo realizado no Sergipe (REINALDO et al., 2017) foi avaliada a prevalência de hipertensão arterial sistêmica (HAS) de uma UAN com 174 trabalhadores, sendo $83,3 \%$ dos pesquisados homens. Verificou-se que $12,1 \%$ dos avaliados eram hipertensos, sendo que 90,4\% também apresentaram sobrepeso. A prevalência de HAS foi de 12,1\%, com ocorrência maior entre os homens (12,4\%). Os pratos proteicos foram o que mais influenciaram a ingestão de sódio, que ficou acima das recomendações do PAT, chegando à média de $2311 \mathrm{mg}$.

Os resultados encontrados no presente estudo revelaram que o consumo de sódio excedeu o valor diário recomendado pelo PAT para as principais refeições. Na refeição do almoço, considerando apenas o sódio utilizado nas preparações, a UAN 1 atingiu $80 \%$ da recomendação diária de sódio estabelecida pelo PAT, que é de $2400 \mathrm{mg}$, e ultrapassou 201\% (968,4 mg) do recomendado para apenas uma refeição principal, que é de no máximo $960 \mathrm{mg}$. A UAN 2 ultrapassou 21\% $(510,8 \mathrm{mg})$ da recomendação diária de sódio estabelecida pelo PAT e 303\% (1950,8 mg) do recomendado para apenas uma refeição principal. Quando contabilizado junto à quantidade de sódio consumida no buffet, os valores aumentam ainda mais.

O PAT vem se destacando quando relacionado ao interesse na promoção de estilos de vida saudáveis de trabalhadores, isto, pois o atual cenário epidemiológico do crescente avanço das doenças crônicas e obesidade no ambiente de trabalho vem ganhando destaque por ser um local propício às modificações de comportamento precursor de doenças, estreitamente relacionadas ao estilo de vida, dieta, atividade física e ao tabagismo (ORO; HAUTRIVE, 2015). 
Estudo realizado por Klein e Silva (2012), em uma cidade do Vale do Taquari, identificou que a ingestão média de sódio nas refeições foi de $3120 \mathrm{mg}$, assim como no presente estudo, ultrapassando o limite recomendado de ingestão desse mineral. No estudo de Klein foi realizada uma campanha com o intuito de reduzir a quantidade ingerida de sal, que foi responsável por reduzir $23 \%$ do consumo de sódio em relação ao habitual.

Para diminuir o consumo de sódio de um determinado grupo, são necessários esforços em larga escala para reduzir o teor de sal dos produtos alimentícios no momento da produção, bem como para iniciar mudanças comportamentais (TEMME et al., 2017). Estudo analisou a frequência de consumo de alimentos marcadores de dieta saudável e não saudável entre homens e mulheres adultos com base nos dados do Sistema de Vigilância de Fatores de Risco e Proteção para Doenças Crônicas não Transmissíveis por Inquérito Telefônico (VIGITEL) e constataram que 19,5\% dos homens consumiam teor alto ou muito alto de sal contra $16,7 \%$ das mulheres (DURANTE et al., 2017).

O sal adicionado durante o cozimento não é a principal fonte de ingestão de sódio. Em muitos países, cerca de $80 \%$ do sal na dieta vem de alimentos processados (WHO, 2016). Brito e Spinelli (BRITO; SPINELLI, 2016) em seu estudo, analisaram o teor de sódio contido nos alimentos industrializados utilizados nas preparações fornecidas por quatro UANs. Em valores médios, a primeira unidade ofereceu 1583,05 mg de sódio em um almoço, a segunda unidade ofertou $970,68 \mathrm{mg}$, a terceira unidade $1.075,05 \mathrm{mg}$ e a quarta unidade 1769,21. Considerando que o PAT estima uma variação de 720 a $960 \mathrm{mg}$ de sódio para refeições principais, a oferta desse mineral é maior do que o recomendado para uma refeição do almoço, principalmente quando é levado em conta que o sal adicionado na preparação das refeições e o sal consumido no buffet não foi contabilizado. É importante salientar que os valores encontrados no presente estudo poderiam aumentar ainda mais se levássemos em conta o sódio oriundo dos alimentos industrializados utilizados nas preparações.

Estima-se que 2,5 milhões de mortes poderiam ser evitadas a cada ano se o consumo global de sal fosse reduzido ao nível recomendado (BRASIL, 2014). O comportamento alimentar é um conjunto de ações relacionadas ao alimento, que envolve desde a escolha até a ingestão, bem como tudo a que ele se relaciona. Por isso, a mudança de hábitos é uma tarefa bastante difícil e complexa, pois envolve além de muito estudo e pesquisa, a própria formação do profissional. É neste aspecto que se evidencia a necessidade de uma intervenção, não só no aspecto prático do que comer ou como comer, mas na questão cultural e até educacional. Existem UANs que trabalham com programas que pretendem promover a saúde dos comensais, e por isso desenvolvem campanhas que vão desde a educação nutricional até a implementação de novas opções no cardápio para incentivar ou desestimular o consumo de alguns tipos de alimentos (BRITO; SPINELLI, 2014).

Como fatores limitadores do presente estudo, é importante ressaltar que na análise das fichas técnicas foi considerada a oferta para os trabalhadores dos diferentes alimentos que os compunham, não levando em conta desperdícios e sobras. Ainda, a UAN é um estabelecimento do tipo self-service, o qual não garante que o comensal se sirva de todos os tipos de preparações que the foi 
disponibilizado. Para resultados mais exatos seria necessário acompanhar durante um maior período de tempo os alimentos ingeridos no almoço pelos comensais, incluindo a sobra de comidas no buffet, sobra limpa e resto ingesta.

Em contrapartida, o estudo se mostra relevante, pois percebeu-se um elevado consumo de sódio na amostra, o que pode refletir no desenvolvimento de patologias como a hipertensão arterial. Ainda, trabalhar educação nutricional entre os comensais, ou mesmo proporcionar o conhecimento em relação aos resultados aos profissionais responsáveis por UANs pode ser um destaque do trabalho para com o incentivo em relação à promoção à saúde de seus comensais e trabalhadores.

\section{CONCLUSÃO}

Embora o sódio seja um mineral importante e necessário para a manutenção da vida, seu consumo em excesso pode contribuir para 0 aparecimento e agravamento de doenças crônicas não transmissíveis. Nesse contexto, torna-se relevante analisar estratégias ligadas às Unidades de Alimentação e Nutrição com o objetivo de formular cardápios que atendam a coletividade, favorecendo a adoção de práticas alimentares saudáveis.

Por meio dos resultados deste estudo, pode-se verificar que as refeições servidas nas duas Unidades de Alimentação e Nutrição não se encontram adequadas às necessidades nutricionais dos seus clientes de acordo com os parâmetros estabelecidos pelo Programa de Alimentação do Trabalhador. Sugere-se reduzir a quantidade de sódio ofertada a fim de contribuir para uma alimentação mais saudável. Visto que para muitos funcionários, a alimentação recebida na empresa representa a grande refeição do dia, a realização de uma correta intervenção alimentar pode representar um importante reflexo na saúde dos comensais, além de estimular a adoção de hábitos alimentares saudáveis.

\section{REFERÊNCIAS}

BALTAZAR, L.; CONDE, S. R.; ALVES, M. K. Teor de sódio no cardápio oferecido a trabalhadores de um hipermercado do interior do Rio Grande do Sul. Revista UNINGÁ, v. 56, n. 4, p. 187-194, 2019.

BEZERRA I. N. et al. Consumo de alimentos fora do lar no Brasil segundo locais de aquisição. Revista de Saúde Pública, v. 51, n. 15, p. 1-8, 2017.

BORGES S. K. S.; CONDE, S. R. Avaliação da quantidade de sódio do cardápio de uma Unidade de Alimentação e Nutrição Hospitalar de Caxias do Sul, RS. Revista UNINGÁ, v. 21, n. 2, p. 10-13, 2015.

BRASIL. Alimentação Cardioprotetora: manual de orientações para profissionais de saúde da Atenção Básica. Brasília: MS, 2018.

BRITO, A. M. O.; SPINELLI M. G. N. Oferta de sódio oriundo de alimentos industrializados em restaurantes institucionais. DEMETRA: Alimentação, Nutrição \& Saúde, v. 11, n. 2, p. 321-336, 2016. 
CARNEIRO, N. S.; MOURA, C. M. A.; SOUZA, S. C. C. Avaliação do almoço servido em uma Unidade de Alimentação e Nutrição, segundo os critérios do programa de alimentação do trabalhador. Alimentos e Nutrição, v. 24, n. 3, p. 361-365, 2013.

CONCEIÇÃO, K. N. et al. Evaluation of consumption of sodium dining offered in central market town. ReonFacema, v. 2, n. 3, p. 229-231, 2016.

OLIVEIRA, M. M. et al. Consumo elevado de sal autorreferido em adultos: dados da Pesquisa Nacional de Saúde, 2013. Epidemiologia e Serviços de Saúde, v. 25, n. 2, p. 249-256, 2016.

REPÚBLICA FEDERATIVA DO BRASIL. Portaria interministerial $\mathrm{n} \times \mathbf{6 6}$, de 25 de agosto de 2006. Diário Oficial da União, Brasília, de 28 de agosto de 2006.

DURANTE, G. D. et al. Diferenças no consumo de alimentos entre homens e mulheres entrevistados pelo Inquérito Telefônico Vigitel. Revista Brasileira em Promoção da Saúde, v. 30, n. 3, p. 1-12, 2017.

INSTITUTO BRASILEIRO DE GEOGRAFIA E ESTATÍSTICA. Pesquisa de orçamentos familiares, 2008-2009: análise do consumo alimentar pessoal no Brasil. IBGE: Rio de Janeiro, 2018.

KLEIN, C.; SILVA A. B. G. Avaliação do consumo de sódio em uma unidade de alimentação e nutrição do Vale do Taquari. Revista Destaques Acadêmicos, v. 4, n. 3, p. 73-78. 2012

LAGEMANN, M. D.; FASSINA, P. Avaliação da composição nutricional de refeições oferecidas em uma Unidade de Alimentação e Nutrição. Caderno Pedagógico, v. 12, n. 1, p. 36-45, 2015.

LOPES, F. S.; LIMA, V. M.; MARTIONS, F. A. Avaliação da adequação nutricional das refeições distribuídas no restaurante popular de Rio Branco, AC. Journal of Amazon Health Science, v. 2, n. 2, p. 1-22, 2016.

MARTINS, W. O. et al. Consumo alimentar e hábitos de vida de hipertensos atendidos em um ambulatório de nutrição de pelotas-rs. Revista Brasileira de Obesidade, Nutrição e Emagrecimento, v. 14, n. 85, p. 273-281, 2020.

MAZZON, J. F. 40 anos do PAT - Programa de Alimentação do Trabalhador: conquistas e desafios da política nutricional com foco em desenvolvimento econômico e social. São Paulo: Blucher, 2016.

MELLO, A. V. et al. Prevalência de Fatores de Risco Cardiovascular Entre Homens e Mulheres Participantes de um Evento de Promoção da Saúde. Ensaios e Ciência, v. 24, n. 1, p. 59-64, 2020.

OLIVEIRA, E. M.; CARVALHO, A. C. S. Condições de trabalho e saúde dos trabalhadores de uma unidade de alimentação e nutrição. Revista Brasileira de Assuntos Interdisciplinares, v. 1, n. 1, p. 43-56, 2017. 
ORO, G. L.; HAUTRIVE, T. P. Avaliação do cardápio do almoço oferecido à trabalhadores atendidos pelo Programa de Alimentação do Trabalhador. eScientia, v. 8, n. 1, p. 1-7, 2015.

OTTONI, I. C.; SPINELLI, M. G. N. Oferta de sódio em refeições de unidades de alimentação e nutrição escolar. Revista Univap, v. 20, n. 35, p. 35-43, 2014.

PEREIRA, J. P. et al. Qualidade das refeições oferecidas por empresas cadastradas pelo Programa de Alimentação do Trabalhador na cidade de Santos-SP. O Mundo da Saúde, v. 38, n. 3, p. 325-333, 2014.

PEREIRA, E. J.; SOUSA, E. F.; RIBEIRO, S. S. S. Análise da adequação dos cardápios de almoço de dois restaurantes de acordo com o recomendado pelo Programa de Alimentação do Trabalhador (PAT). Revista Nutrire, v. 36, n. 11, p. 142-142, 2011.

REINALDO, J. M.; RESENDE, A. S.; SANT ANNA, M. S. L. Prevalência de hipertensão arterial e avaliação da ingestão de sódio em uma Unidade de Alimentação e Nutrição do Estado de Sergipe/Brasil. Revista da Associação Brasileira de Nutrição, v. 8, n. 1, p. 58-63, 2017.

SALAS, C. K. T. S. et al. Teores de sódio e lipídios em refeições almoço consumidas por trabalhadores de uma empresa no município de Suzano, SP. Revista de Nutrição, v. 22, n. 3, p. 331-339, 2009.

SPINELLI, M. G. N.; KAWASHIMA, L. M.; EGASHIRA, E. M. Análise de sódio em preparações habitualmente consumidas em restaurantes self-service. Alimentação Nutrição, v. 22, n. 1, p. 55-61, 2011.

STEFFENSEN, I. L. et al. Benefit and risk assessment of increasing potassium intake by replacement of sodium chloride with potassium chloride in industrial food products in Norway. Food and Chemical Toxicology, v. 11, p. 329-340. 2018

TEMME, E. H. M. et al. Salt Reductions in Some Foods in The Netherlands: Monitoring of Food Composition and Salt Intake. Nutrients, v. 9, n. 7, p. 791, 2017.

TURNER, L. R. et al. Cardiovascular disease screening in general practice: General practitioner recording of common risk factors. Preventine Medicine, v. 99, p. 282-285, 2017.

VAZ, D. S. S.; BENNEMANN, R. M. Comportamento alimentar e hábito alimentar: Uma revisão. Revista UNINGÁ, v. 20, n. 1, p. 108-112, 2014.

WORLD HEALTH ORGANIZATION. Salt reduction. 2016. Acesso em: 07 set. 2018. Disponível em: http://www.who.int/news-room/fact-sheets/detail/saltreduction. 